\title{
Conformal properties of charges in scalar-tensor gravities
}

\author{
S Deser ${ }^{1,2}$ and Bayram Tekin ${ }^{3}$ \\ ${ }^{1}$ Department of Physics, Brandeis University, Waltham, MA 02454, USA \\ 2 Lauritsen Lab, Caltech, Pasadena CA 91125, USA \\ ${ }^{3}$ Department of Physics, Faculty of Arts and Sciences, Middle East Technical University, 06531, \\ Ankara, Turkey \\ E-mail: deser@brandeis.edu and btekin@metu.edu.tr
}

Received 26 September 2006, in final form 14 October 2006

Published 13 November 2006

Online at stacks.iop.org/CQG/23/7479

\begin{abstract}
We study the behaviour under conformal transformations of energy and other charges in generic scalar-tensor models. This enables us to conclude that the $\mathrm{ADM} / \mathrm{AD}$ masses are invariant under field redefinitions mixing metric and scalar despite the permitted slow asymptotic falloff of massless scalars.
\end{abstract}

PACS numbers: $04.20 . \mathrm{Cv}, 04.50 .+\mathrm{h}$

\section{Introduction}

Gravitational models involving massless scalars and (possibly) a cosmological constant can cause complications in applying otherwise well-understood energy and other definitions of physical quantities. In part, this is due to the scalars' slow allowed asymptotic falloff, generically as slow as that of the metric, $1 / r^{D-3}$, along with the possibility of scalartensor field redefinitions, $g_{\mu \nu} \rightarrow g_{\mu \nu} f(\phi)$. One well-know example of deviation from pure Einstein behaviour is the violation of the equivalence principle: the coefficients of the leading asymptotic terms of $g_{00}$ and $g_{i j}$ become unequal; the former define the Newtonian force, the latter the system's energy. Here, the potential difficulty is that, while energy for asymptotically flat (ADM [1]) or asymptotically (A)dS [2-4] spaces is defined as though scalars are just another form of matter, their formal role can be shifted by the above field redefinitions that do not alter the physics any more than any other such field redefinitions in field theories ${ }^{4}$. We show here that indeed the correct physical quantities are invariant as desired, if not transparently so.

4 The conformal invariance of the distinct, if related, quasi-local mass has been previously established in [5]. 


\section{The generic action}

The most general second-order scalar-tensor theory in $D$ dimensions involves four arbitrary functions of the scalar field,

$S=\frac{1}{2 \kappa} \int \mathrm{d}^{D} x \sqrt{-g} U(\phi)\left\{R(g)+2 \Lambda_{0}-W(\phi) \partial_{\mu} \phi \partial^{\mu} \phi-V(\phi)+H(\phi) \mathcal{L}_{\mathrm{m}}\right\}$,

where $\mathcal{L}_{\mathrm{m}}$ represents all the matter besides the scalar. One-standard-field redefinition to the Einstein frame removes the overall function $U(\phi)$

$$
g_{\mu \nu}^{E} \equiv U(\phi)^{\frac{2}{D}} g_{\mu \nu}
$$

leading to

$$
S=\frac{1}{2 \kappa} \int \mathrm{d}^{D} x \sqrt{-g^{E}}\left\{R\left(g^{E}\right)+2 \Lambda_{0}\right\}+S_{M}
$$

where $S_{M}$ now has the form

$$
\left.S_{M}=\frac{1}{2 \kappa} \int \mathrm{d}^{D} x \sqrt{-g^{E}}\left\{A(\phi) \partial_{\mu} \phi \partial^{\mu} \phi+X(\phi)+Z(\phi) \mathcal{L}_{\mathrm{m}}\right]\right\} .
$$

Obviously, a rescaling of $\phi$ reduces its kinetic term to free form, leaving just two arbitrary functions, the scalar self-interaction and a possible coefficient of the matter action. We emphasize that this describes the same physics as (1) via a different metric variable. But the conserved and diffeo-invariant charges of the theory, similarly defined in terms of the metric variables each frame, do not obviously have the same value in each (for the same physical configuration, of course), so one must verify this fact. (One can also study other conformal properties of the spacetimes. For example in [6] it was shown that the surface gravity and the temperature of a stationary black hole are invariant under conformal transformations that approach unity at infinity, as is the entropy [7]. These properties, being related to the null horizon, are a priori more likely candidates for conformal invariance than the 'spacelike' mass.)

We have assumed here that the cosmological term is not altered by $V$, i.e. that $V(0)=0$, something that we may always arrange or else just redefine things beforehand. In that case, the UV term would not change the value $\Lambda_{0}$ either, as long as $U(\phi)$ does not rise, $U(0)=$ const. $^{5}$

\section{Conserved charges}

The conserved charges for the action (3) were derived in [2-4] for arbitrary (including vanishing) $\Lambda_{0}$. The result, for any of them (depending on the particular Killing vector), is

$$
\begin{aligned}
Q^{\mu}\left(\bar{g}^{E}, \bar{\xi}^{E}\right)= & \frac{1}{4 \Omega_{D-2} G_{D}} \int_{\partial M} \mathrm{~d} S_{i} \sqrt{\bar{g}^{E}}\left\{\bar{\xi}^{E}{ }_{\nu} \bar{\nabla}^{\mu} h_{E}^{i \nu}-\bar{\xi}^{E}{ }_{\nu} \bar{\nabla}^{i} h_{E}^{\mu \nu}+\bar{\xi}_{E}^{\mu} \bar{\nabla}^{i} h^{E}-\bar{\xi}_{E}^{i} \bar{\nabla}^{\mu} h^{E}\right. \\
& \left.+h_{E}^{\mu \nu} \bar{\nabla}^{i} \bar{\xi}^{E}{ }_{\nu}-h_{E}^{i \nu} \bar{\nabla}^{\mu} \bar{\xi}^{E}{ }_{\nu}+\bar{\xi}_{E}^{i} \bar{\nabla}_{\nu} h_{E}^{\mu \nu}-\bar{\xi}_{E}^{\mu} \bar{\nabla}_{\nu} h_{E}^{i v}+h^{E} \bar{\nabla}^{\mu} \bar{\xi}_{E}^{i}\right\}
\end{aligned}
$$

where we have split $g_{\mu \nu}^{E} \equiv \bar{g}_{\mu \nu}^{E}+h_{\mu \nu}^{E}$ and defined $h^{E}=h_{\mu \nu}^{E} \bar{g}_{E}^{\mu \nu} ; \bar{\xi}^{E}$ is a Killing vector with respect to the asymptotic (A)dS or flat background $\bar{g}_{E}$. The integral is to be computed on a spatial hypersurface at infinity (only then is the expression diffeomorphism invariant). The covariant derivatives are also with respect to the Einstein-frame metric. For flat background,

\footnotetext{
5 A second issue is one mentioned in [8,9], namely possible scalar configurations whose kinetic and potential terms make divergent contributions to the energy. This would require a more careful choice of background with respect to which the energy is to be measured.
} 
(5) reduces to the ADM mass [1], but in arbitrary, rather than the Cartesian coordinates. If the background is $\mathrm{dS}$, then as is well-understood, the cosmological horizon forbids timelike Killing vectors outside, and one can only deal with systems localized within the horizon.

To return to our energy problem, consider the inverse transformation to (2), from Einstein to generic frame. This leads to the following scaling of the integrand of the conserved charge (5)

$$
\begin{gathered}
\sqrt{g} q^{i \mu}(\xi)=U^{-\frac{2}{D}} \sqrt{\bar{g}^{E}}\left\{q^{i \mu}\left(\xi^{E}\right)-\frac{3}{D} \xi_{v}^{E} h_{E}^{i \nu} \partial^{\mu} \log U+\frac{3}{D} \xi_{v}^{E} h_{E}^{\mu \nu} \partial^{i} \log U\right. \\
\left.-\frac{D-1}{D} \xi_{E}^{i} h_{E}^{\mu \nu} \partial_{\nu} \log U+\frac{D-1}{D} \xi_{E}^{\mu} h_{E}^{i \nu} \partial_{\nu} \log U\right\}
\end{gathered}
$$

and immediately proves our desired result: if $U(\infty)=1$ then $g_{\mu \nu}$ and $g_{\mu \nu}^{E}$ have the 'same' charges. If, on the other hand, $U(\infty)$ is some arbitrary constant, then the charges of these two metrics differ by a multiplicative constant. This result means that all charges given in the form (5) are invariant.

Our construction is quite generic: higher curvature models, to which we now turn , can also be handled in a similar fashion as above. In [3, 4], we constructed conserved charges in generic higher curvature gravity models. First let us consider the theories known as $F(R)$ gravities recently suggested in connection with the accelerated expansion of the Universe. Their actions read

$$
S=\frac{1}{2 \kappa^{2}} \int \mathrm{d}^{4} x \sqrt{-g}\left(F(R)+2 \Lambda_{0}\right)+S_{m},
$$

where $F(R)$ is a function of the Ricci scalar $R$ only. For example, it could be $R+\mu^{4} / R$.

The model (7) can be re-expressed as a scalar-tensor theory

$$
S=\frac{1}{2 \kappa^{2}} \int \mathrm{d}^{4} x \sqrt{-g}\left(F(\phi)+F^{\prime}(\phi)(R-\phi)+2 \Lambda_{0}\right)+S_{\text {matter }},
$$

where $F^{\prime}(\phi)=\mathrm{d} F / \mathrm{d} \phi$ and $F^{\prime \prime}(\phi) \neq 0$. Furthermore, assuming that $F^{\prime}(\phi) g_{\mu \nu}=g_{\mu \nu}^{E}$ the action can be reduced to that of the scalar field minimally coupled to the Einstein gravity. The explicit form of the action is not needed here, as we are only interested in the fact that the conserved charges once again are given as (5).

Lastly, let us consider quadratic models of the form

$$
I=\int \mathrm{d}^{D} x \sqrt{-g}\left\{\frac{R}{\kappa}+\alpha R^{2}+\beta R_{\mu \nu}^{2}+\gamma\left(R_{\mu \nu \rho \sigma}^{2}-4 R_{\mu \nu}^{2}+R^{2}\right)\right\} .
$$

Rescaling of the metric (2) maps the above model to a highly complicated one, whose action we do not need here. But, in the line of the discussion above, let us see how the charges transform under such a scaling. In [3, 4], it was shown that, for the asymptotically AdS spaces, the non-trivial part of the charge is given as

$$
Q^{\mu}=\left\{\frac{1}{\kappa}+\frac{4 \Lambda D \alpha}{D-2}+\frac{4 \Lambda \beta}{D-1}+\frac{4 \Lambda \gamma(D-4)(D-3)}{(D-2)(D-1)}\right\} \times Q_{\text {Einstein }}^{\mu}
$$

where $Q_{\text {Einstein }}^{\mu}$ is given by (5). Therefore, the conserved charges of the theory (9) transform as in (6) under conformal scalings of the metric. Any higher curvature model, including actions that depend on inverse powers of the scalar invariants constructed from the Ricci and Riemann tensors, can be handled this way. But, of course, getting the surface form of the energy expressions will be tricky in some cases. 


\section{Summary}

We have studied the properties of conserved charges in various gravity models, such as Einstein, higher curvature models and scalar-tensor theories, both for asymptotically AdS and flat spacetime: they are conformally invariant as long as the conformal factor goes to unity at infinity. Our formalism also relates the charges of a generic scalar-tensor theory to Einstein's theory minimally coupled to scalar fields. Amongst other open problems, we hope to return to the treatment of solutions with scalar fields that radically alter the asymptotics.

\section{Acknowledgments}

The work of SD is supported by NSF grant PHY 04-01667, that of BT by the 'Young Investigator Fellowship' of Turkish Academy of Sciences (TUBA) and by the TUBITAK Kariyer grant no $104 \mathrm{~T} 177$. We thank a referee for pointing out $[5,7]$ and for suggesting our comments regarding the latter.

\section{References}

[1] Arnowitt R, Deser S and Misner C W 1960 The dynamics of general relativity Phys. Rev. 1171595 Arnowitt R, Deser S and Misner C W 1962 The dynamics of general relativity Gravitation: An Introduction to Current Research ed L Witten (New York: Wiley) (reprinted as gr-qc/0405109)

[2] Abbott L F and Deser S 1982 Stability Of Gravity With A Cosmological Constant Nucl. Phys. B 19576

[3] Deser S and Tekin B 2002 Gravitational energy in quadratic curvature gravities Phys. Rev. Lett. 89101101

[4] Deser S and Tekin B 2003 Energy in generic higher curvature gravity theories Phys. Rev. D 67084009

[5] Bose S and Lohiya D 1999 Behavior of quasilocal mass under conformal transformations Phys. Rev. D 59044019

[6] Jacobson T and Kang G 1993 Conformal invariance of black hole temperature Class. Quantum Grav. 10 L201

[7] Ashtekar A and Corichi A 2003 Non-minimal couplings, quantum geometry and black hole entropy Class. Quantum Grav. 204473

[8] Henneaux M, Martinez C, Troncoso R and Zanelli J 2004 Asymptotically anti-de Sitter spacetimes and scalar fields with a logarithmic branch Phys. Rev. D 70044034

[9] Chen W, Lu H and Pope C N 2006 Mass of rotating black holes in gauged supergravities Phys. Rev. D 73104036 